\title{
Shear Swelling of Polymer Brushes Grafted onto Convex and Concave Surfaces
}

\section{E. M. Sevick}

Research School of Chemistry, Institute of Advanced Studies, The Australian National University, Canberra ACT 0200, Australia

Received March 25, 1996; Revised Manuscript Recei ved J une 24, $1996^{\otimes}$

\begin{abstract}
The shear response of polymer brushes is explored as a function of grafting surface curvature using the Alexander-deGennes ansatz, where the free ends of the chains are localized at the tip of the brush and all tethered chains in the brush are stretched equally. Brushes adsorbed onto the concave and convex surfaces of a cylinder are found to swell to a maximum of $35 \%$ of their nonsheared brush height in good solvent, larger than the predicted maximum shear swelling of planar brushes of the same grafting density. For significantly convex surfaces, increased surface curvature increases the maximum amount of shear swelling. In contrast, brushes grafted onto concave surfaces with the same grafting density show diminished shear swelling as the grafting surface becomes more concave. The shear response of nonplanar brushes is important in a variety of possible applications, including the fabrication of pressure-sensitive microvalves and dynamic membranes and an understanding of the rheol ogical behavior of hairy-rod polymers or molecular bottlebrushes.
\end{abstract}

\section{Introduction}

The physical properties of polymer brushes grafted onto surfaces is important in the modification of surfaces and the stabilization of colloidal suspensions. A polymer brush consists of a high density of polymers end-grafted onto a surface. The polymers are grafted so densely that they overlap strongly and are forced to stretch away from the surface, forming an elastic layer. Recently, experiment ${ }^{1,2}$ and theory ${ }^{3-5}$ have focused on the response of such brushes to an applied tangential surface force. Klein et al.1,2 demonstrated that a pair of opposing brushes, when slid past one another in the presence of a good solvent, experience a repulsive normal force and interpreted this as brush swelling under shear. This novel shear response has spurred speculation on new applications of polymer brushes, beyond the usual application of colloidal stabilization. Pressure-sensitive microvalves constructed from brushlined conduits were proposed:6,7 the brush senses the shear force, responds by swelling, and thereby readjusts the shear and flow, much like a system of sensors and valves. A porous substrate lined with polymer brush has also been proposed for modulation of flow-through porous media. ${ }^{8,9}$ The shear response of brushes may also be important to the rheological behavior of hairyrod polymers ${ }^{10,11}$ or molecular bottlebrushes:12,13 these bottlebrush molecules consist of a relatively stiff main chain from which flexible side chains emanate. We predict that these molecules swell when sheared, making the mol ecule larger in shearing fields. To investigate these and other potential applications, we need to determine the shear response of brushes tethered not only to planar surfaces but also to convex and concave ones. How does the shear response of the brush depend on the curvature of the grafting surface?

In the absence of shear, the height of brushes grafted onto planar and convex surfaces is understood from theory, simulation, and experiment to be a result of the balance between the osmotic pressure (which tends to swell the layer) and the elasticity of the tethered chains (which opposes swelling). The simplest description, \footnotetext{
1996.

${ }^{\otimes}$ Abstract published in Advance ACS Abstracts, August 15,
}

using the Alexander-deGennes ansatz, ${ }^{14}$ shows that the height of a planar brush scales as $\mathrm{H}_{0} \propto \mathrm{Nd}^{-2 / 3}$, where $\mathrm{N}$ is the degree if polymerization and $1 / \mathrm{d}^{2}$ is the grafting density or $d$ the distance between grafting points. Daoud and Cotton ${ }^{15}$ extended the free energy scaling approach to polymers end-tethered onto a spherical grafting surface with vanishing radius, and Birshtein et al. ${ }^{16}$ considered grafting onto a convex surfaces, predicting the brush height, $\mathrm{H}_{0}$ to scale as

$$
\mathrm{H}_{0} \equiv \mathrm{aN}^{3 /(3+\mathrm{D})} \mathrm{d}^{-2 /(3+\mathrm{D})}(\mathrm{R} / \mathrm{a})^{\mathrm{D} /(3+\mathrm{D})}
$$

where $\mathrm{D}=0,1$, or 2 for planar, cylindrical, or spherical surfaces, respectively, with the brushes adsorbed onto a convex surface of radius $R$. These scaling treatments used the Alexander-deGennes ansatz, which restricted all free chain ends to be located at the edge of the brush and all tethered chains to be stretched identically. More detailed treatment of chains tethered to both planar and curved surfaces indicates that the free ends are distributed within a brush and that the distribution depends on the curvature of the surface. These advanced theor etical methods, applied to curved surfaces, include Monte Carlo and molecular dynamics simulations ${ }^{17,18}$ and self-consistent field (SCF) calculations. ${ }^{19,22}$ Neutron reflectivity measurements ${ }^{23}$ and small-angle neutron scattering ${ }^{24}$ verify the predictions of chain end and monomer density profiles within planar brushes. While these analyses provide detailed descriptions of the brush profile, all demonstrate that the Alexander-de Gennes ansatz correctly describes the scaling of the most readily measured feature of planar and convex brushes: their height.

In contrast, the height of a polymer brush upon subjecting the brush to a tangential shearing force is a subject only recently investigated, starting with the surface force measurements of Klein et al. ${ }^{1,2}$ Using a free energy model originally proposed by Rabin and Alexander, ${ }^{4}$ Barrat $^{3}$ considered an AlexanderdeGennes brush and showed theoretically that the swelling can be the result of an imbalance in the osmotic and elastic components of the free energy caused by the shear-induced stretching of the tethered chains. Barrat's analysis predicted a planar brush swelling which grows smoothly with surface shearing force from $0 \%$ to 
a maximum of $25 \%$ of the no-shear brush height, which reasonably describes the brush expansion in the experiments. ${ }^{1,2}$ However, computer simulation studies of sheared, grafted chains do not recover the shear swelling of experiment and scaling theory. Lai and Binder ${ }^{25}$ used nonequilibrium Monte Carlo simulation with a bond fluctuation model and solvent flow profile calculated using Gaussian chains, and Peters and Tildesley ${ }^{26}$ reported on nonequilibrium molecular dynamics simulation of tethered 20-mer chains surrounded by solvent molecules. While neither simulation study reproduced brush swelling, it is not evident that the simulations provide definitive evidence against brush swelling. This is essentially because each simulation used a small number of monomers per chain.

In this article, we study the shear behavior of a brush adsorbed onto the interior or exterior surface of a cylinder with an imposed tangential shear force acting at the brush tips (Figure 1). Our description is limited to the case of good solvents where brush swelling, rather than brush compression, occurs with shear. ${ }^{27}$ The simplest model of brush structure is adopted in the free energy analysis: that of a brush whose free ends are localized at the tip of the brush and where all chains possess the same stretching profile. This approximation is anal ogous to the Alexander-deGennes approximation of a step function profile for planar brushes; consequently, we will hereafter refer to it as an AlexanderdeGennes ansatz. The Alexander-deGennes assumption is good for brushes grafted onto convex surfaces but becomes increasingly poor for concave brushes. We find that the brush response to shear depends on the convexity of the grafting surface, exhibiting an increased response to shear with an increase in convexity. In contrast, concave brushes swell nonmonotonically with shearing force and become insensitive to shear in highly concave geometries. The remainder of the paper is organized in the following manner. In section II, the free energy analysis ${ }^{3,4}$ is briefly reviewed and extended to account for grafting surface curvature. The resulting free energy equation is then used to predict no-shear brush height as a function of grafting surface convexity and concavity, which is compared with previous predictions. In section III, we describe the results of the free energy scaling with an imposed shearing force, predicting swelling behavior as a function of applied shearing force and grafting surface curvature. Section IV concludes with a discussion of results and a comparison of the results with the free energy gradients of a simplified model from which the nonmonotonic swelling of concave brushes and monotonic swelling of planar and convex brushes can be easily understood.

\section{Free Energy Model}

To construct the free energy for a brush grafted to a cylindrical surface, we first consider a planar brush.3,4 The grafting density of chains in the brush is $1 / \mathrm{d}^{2}$, where $\mathrm{d}$ is the distance between grafting sites, and there are $\mathrm{N}_{\mathrm{c}}$ chains in the brush. Each chain in the planar brush can be pictured as a connected sequence of Pincus blobs. 28,29 The blobs are of radius $\xi=\mathrm{R}_{F}{ }^{5 / 2} / \mathrm{L}^{3 / 2}$, where $\mathrm{R}_{\mathrm{F}}=\mathrm{aN}^{3 / 5}$ is the Flory radius and $\mathrm{L}$ is the end-to-end distance of the chain or, equivalently, the height of the brush. Within each blob, the monomers are locally correlated as in a Flory excluded-vol ume chain, but for larger length scales of $\xi$, the blobs behave as hard spheres. The number density of blobs per chain is $\mathrm{N}_{\mathrm{b}}$ $=\left(L / R_{F}\right)^{5 / 2}$. The height, $H_{0}=L$, is found by minimiza-
A

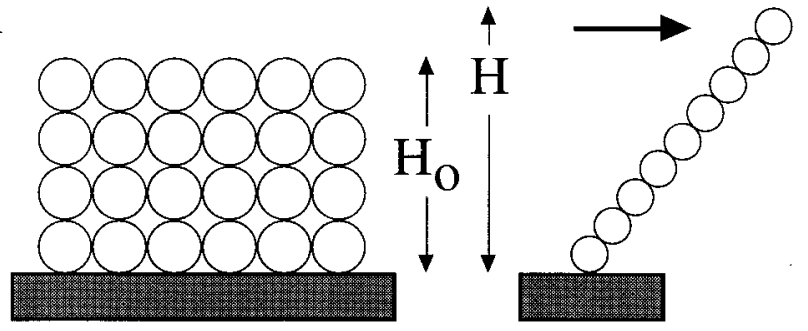

B

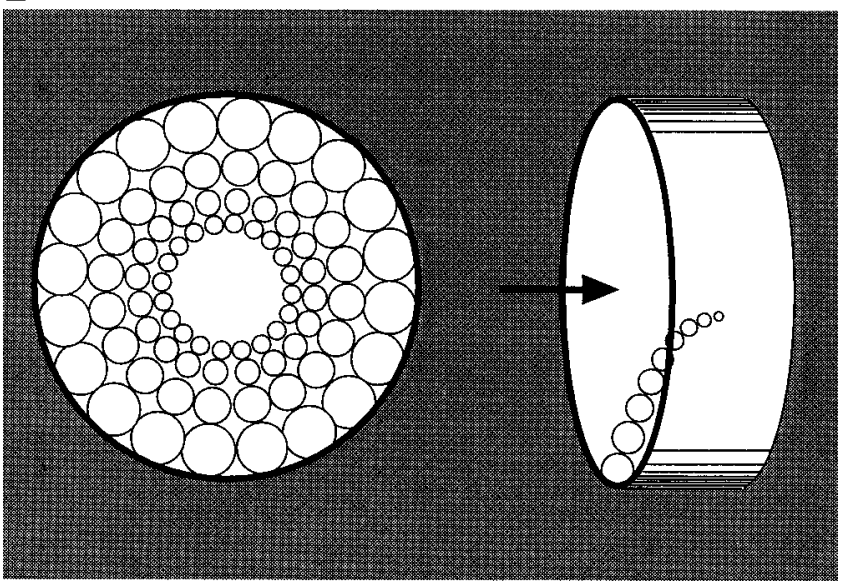

C

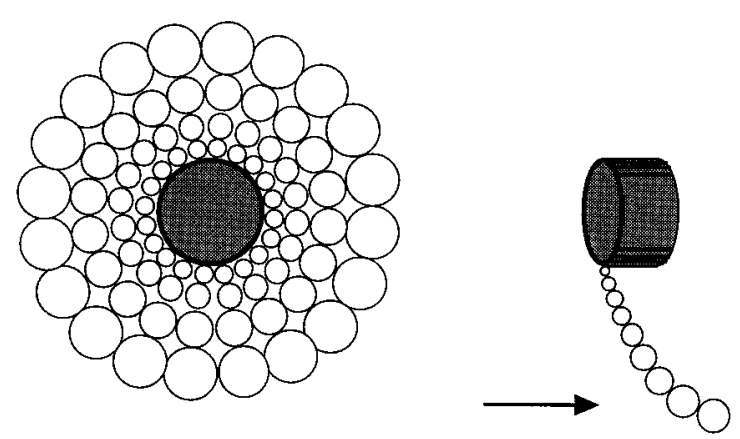

Figure 1. Schematic of polymer brush grafted onto (A) a planar surface, (B) the concave (or interior) surface of a cylinder, and (C) the convex (or exterior) surface of a cylinder. All chains are depicted in accord with the AlexanderdeGennes description, where all chains in the brush are stretched identically, and the free ends of the tethered chain detail the no shear height of the brush, $\mathrm{H}_{0}$. In the planar case, the chains are uniformly stretched, and the monomers are distributed in a step profile. In the curved surfaces, each tethered chain is stretched nonuniformly, as indicated by the variation in blob size along the contour of each tethered chain. Concave tethered chains are more locally stretched at the tip of the brush; chains tethered to a convex surface are more locally stretched at the root of the brush. Also depicted is an isolated chain in the brush subjected to a shearing force at the tip of the brush, due possibly to flow (A) over the planar brush, (B) inside the cylinder lined with a concave brush, and (C) outside the cylinder lined with a convex brush. In this paper, the effect of grafting surface convexity/concavity upon the shear-induced brush swelling and maximum swelling is described.

tion of the chain energy per chain, $F$, given by the Gaussian stretching penalty of a chain of blobs and the excluded volume interactions between blobs, each of which are uniform within the brush:

$$
\mathrm{F}=\mathrm{kt}\left(\mathrm{L}^{2} / \mathrm{N}_{\mathrm{b}} \epsilon^{2}\right)+2 \mathrm{kT} \epsilon^{3} \mathrm{C}^{2} \mathrm{~V}
$$

where $\mathrm{c}=\mathrm{N}_{\mathrm{b}} \mathrm{N}$ is the concentration of blobs and $\mathrm{V}=$ $d^{2} L$ is the volume per chain. 
Equation 2 may be recast for nonplanar grafting surfaces by noting the variation in stretching and excluded volume energies along the contour of a tethered chain. To account for these variations, the chain is discretized into monomers, labeled 0 through $\mathrm{N}$, where the 0th monomer is equated with the free end and the Nth monomer labels the tethered end. Let $r(m)$ be the location of the mth monomer, measured radially from the center of the cylinder, and $s(m)$ the location of the mth monomer along the chain contour measured from the $m=0$ free end of the chain. In this notation, $r(0)$ is the location of the tip of the brush measured from the cylinder center, and $|r(\mathrm{~N})-r(0)|$ is the brush height, $\mathrm{H}$. The blob size, $\xi(\mathrm{s})$, changes from $\xi=a^{5 / 2} \mathrm{~N}^{3 / 2} \mathrm{~L}^{-3 / 2}$ for a uniformly stretched chain to $\xi(\mathrm{s})=\mathrm{a}^{5 / 2}(\mathrm{dm} / \mathrm{ds})^{3 / 2}$ for the blob located $\mathrm{s}(\mathrm{m})$ along the chain contour. Likewise, the number of blobs along a portion of the contour length $\mathrm{s}$ to $\mathrm{s}+\mathrm{ds}$ is $\mathrm{dN}_{\mathrm{b}}(\mathrm{s})=\mathrm{a}^{-5 / 2}(\mathrm{ds} / \mathrm{dm})^{3 / 2} \mathrm{ds}$. The free energy per chain of the brush is comprised of stretching, excluded vol ume, and shearing components. The stretching energy of the tethered chains is given by the usual Gaussian energy associated with a chain of blobs,

$$
\mathrm{F}_{\text {stretch }}=\mathrm{N}_{\mathrm{c}} \int \mathrm{ds} \frac{\mathrm{kT}}{\mathrm{a}}\left(\frac{1}{\mathrm{a}} \frac{\mathrm{ds}}{\mathrm{dm}}\right)^{3 / 2}
$$

where the integral is over the contour of the chain. The excluded volume energy is

$$
\mathrm{F}_{\text {excluded }}=2 \mathrm{kT} \int \mathrm{dV} \epsilon^{3} \mathrm{c}^{2}
$$

where $\mathrm{c}=\mathrm{N}_{\mathrm{C}} \mathrm{N}_{\mathrm{b}} \mathrm{N}$ is the local concentration of blobs and the integral is over the volume of the brush, and the shearing energy is

$$
\mathrm{F}_{\text {shear }}=\mathrm{N}_{\mathrm{c}} \mathrm{F}_{\text {parallel }} \mathrm{dy}
$$

where dy is the displacement of the free end in the direction of the applied shear, $F_{\text {parallel, }}$ and the shearinduced local tilt angle is $\mathrm{dr}(\mathrm{m}) / \mathrm{ds}(\mathrm{m})=\cos \theta(\mathrm{m})$, as detailed in Figure 2.

We now make two approximations. First, we adopt the Alexander-deGennes ansatz for the nonplanar brush such that the position of the free end, $r(0)$, yields the brush height. The second approximation made in this analysis is that the chain maintains a linear profile, i.e., the tile angle is constant over the chain profile: $\theta(\mathrm{m})$ $=\theta$ for $1<\mathrm{m}<\mathrm{N}$. The expression for the free energy per chain reduces to

$$
\begin{array}{r}
\frac{\mathrm{F}}{\mathrm{LkT}}=\frac{2 \pi \mathrm{R}}{\mathrm{d}^{2}} \int_{0}^{\mathrm{N}} \mathrm{dm}\left(\cos ^{-5 / 2} \theta\left|\frac{\mathrm{dx}}{\mathrm{dm}}\right|^{5 / 2}+\right. \\
\left.\frac{\mathrm{W}}{\mathrm{x}} \cos ^{-1 / 2} \theta\left|\frac{\mathrm{dx}}{\mathrm{dm}}\right|-1 / 2-\mathrm{fa} \sin \theta\left|\frac{\mathrm{dx}}{\mathrm{dm}}\right|\right)
\end{array}
$$

where $\mathrm{W}=2 \mathrm{Ra} / \mathrm{d}^{2}$ and the brush profile is given in units of monomer size, $a$, such that $x(m)=r(m) / a$. The chain stretching, $\mathrm{dx} / \mathrm{dm}$, is expressed as an absolute value so as to permit the use of chains tethered to either concave or convex surfaces without relabeling the monomers.

The free energy expression, eq 6, recovers the previous scaling predictions for nonsheared brushes $(f=0, \cos \theta$ $=1$ ) grafted (1) to a planar surface and (2) to a cylinder with a radius that is small compared with the brush height. The first case, the planar case, occurs in the limit $R \rightarrow \infty$ or $R / r(m) \rightarrow 1$ for $0<m<N$, so that $W / x(m)$ $=(\mathrm{a} / \mathrm{d})^{2} \mathrm{R} / \mathrm{r}(\mathrm{m})=(\mathrm{a} / \mathrm{d})^{2}$. As the stretching profile becomes uniform with less curvature of the grafting

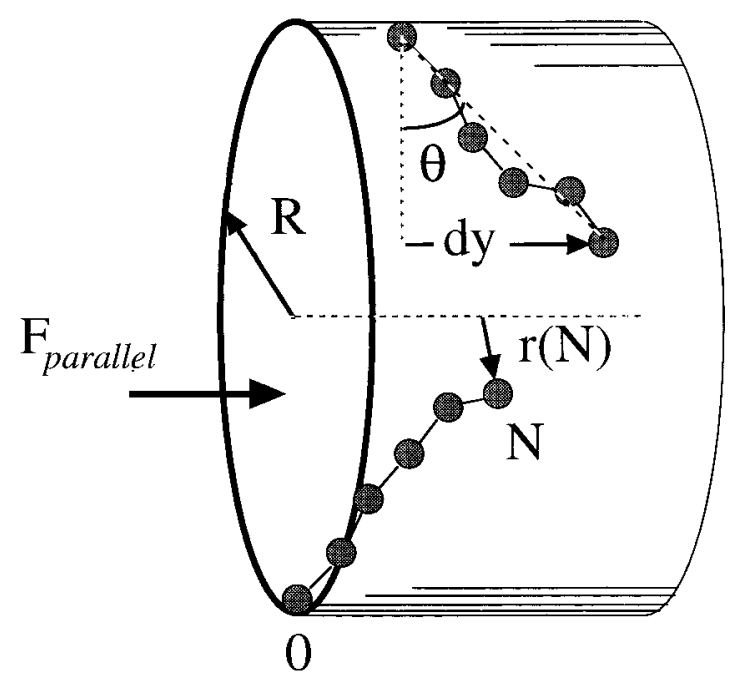

Figure 2. Diagram of the discretization and geometric parameters for a chain tethered to the interior surface of a cylinder with an applied shearing force, $F_{\text {parallel, acting at the }}$ surface of the brush, distorting the brush profile. The chain is discretized into monomers labeled $\mathrm{N}$ at the grafting plane to 0 at the free end, and the location along the contour of the $\mathrm{mth}$ monomer is given by $\mathrm{s}(\mathrm{m})$. The location of the mth monomer measured radially from the center of the cylinder is $r(m)$, such that the brush height is $|r(N)-r(0)|$. The displacement of the tip of the brush due to the shearing force is dy, and the local tilt angle $\theta(\mathrm{m})$ is assumed in this work to be constant over the contour of the chain, rendering a linear chain profile.

surface, $d(a x) / d m \rightarrow H / N$, and minimization of $F$ yields $\mathrm{H}_{0} \sim \mathrm{Nd}^{-2 / 3}$, or eq 1 with $\mathrm{D}=0$. The height of a brush grafted onto the exterior of a cylinder with $\mathrm{R} / \mathrm{H} \rightarrow 0$ (the second case) is recovered by using the simple replace ments $d x(m) / d m=H / N$ and $x=a^{-1}(R \pm H / 2)$. Minimizing over $\mathrm{H}$ yields $\mathrm{H}_{0} \sim \mathrm{N}^{3 / 4}(\mathrm{a} / \mathrm{d})^{1 / 2}(\mathrm{R} / \mathrm{a})^{1 / 4}$, or eq 1 with $\mathrm{D}=1$.

Figure 3 shows the results of the minimization of eq 6 for the height of nonshear brushes grafted onto convex and concave surfaces. The height is found from the profile vector $\mathrm{x}(\mathrm{m})$ for $0<\mathrm{m}<\mathrm{N}-1$, which minimizes the objective function $\mathrm{F}$ with $\theta=0$ using the quasiNewton minimization routine GQBFGS with the BFGS Hessian updating scheme. ${ }^{30}$ Minimization results are independent of discretization of the chain, $100<\mathrm{N}<$ 500 , and the grafting density, $8<\mathrm{d} / \mathrm{a}<12$. The concave and convex brush heights are found for any chosen cylindrical radius, $R$, by appropriate choice of the initial estimate of $x(m)$; i.e., minimization yields the concave brush profile with an initial estimate of $x(0)<x(1)<\ldots$ $<x(N-1)<R / a$, while a convex brush profile is recovered if the initial estimate is $\mathrm{R} / \mathrm{a}<\mathrm{x}(\mathrm{N}-1)<\mathrm{x}$ (N $-2)<\ldots<x(1)<x(0)$. The height of the brush, $H_{0}$, is given by $|\mathrm{R}-\mathrm{ax}(0)|$. In Figure 3 , the height of the brush is scal ed by the height of the brush for a planar surface with the same grafting density, $\mathrm{H} / \mathrm{H}_{\text {planar, where the }}$ subscript zero, denoting no-shear, has been dropped. The radius of curvature is expressed as the radius of the cylinder divided by the brush height, $\mathrm{R} / \mathrm{H}$.

There are clearly two regimes for the convex brush, with crossover around $\mathrm{R} / \mathrm{H}=1$. The brush height in highly convex geometries, $\mathrm{R} / \mathrm{H}<1$, follows the scaling relation of eq 1 , while $R / H>1$ recovers the planar brush. A semi-log presentation of the same data, Figure 5 , emphasizes that, at the crossover curvature, the convex brush height is slightly larger than that of the planar brush, exceeding $\mathrm{H}_{\text {planar }}$ by only $2 \%$. This slight increase in brush height at small curvature, $\delta \mathrm{H}$, follows 


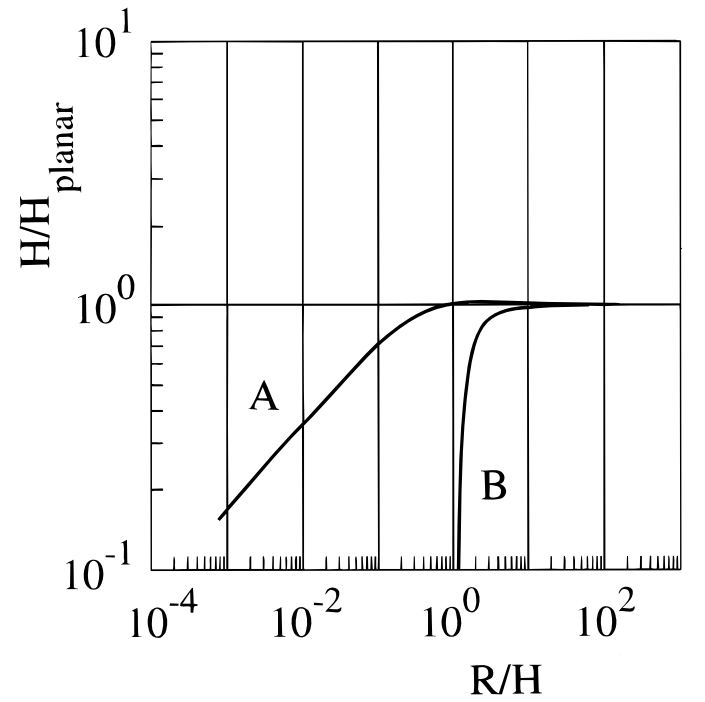

Figure 3. Reduction in brush height, $\mathrm{H} / \mathrm{H}_{\text {planar, as a function }}$ of curvature, $\mathrm{R} / \mathrm{H}$, for a nonshear brush with fixed grafting density $8<$ d/a $<12$ and degree of polymerization $100<\mathrm{N}<$ 500 , found from minimization of eq 6 with $\theta=0$. (A) Brush grafted onto the exterior or convex surface of a cylinder. Two distinct regimes are evident: a convex regime with $\mathrm{H} / \mathrm{H}_{\text {planar }}$ $\sim(\mathrm{R} / \mathrm{H})^{1 / 3}$, in accord with eq 1 , and a planar regime with $\mathrm{H} / \mathrm{H}_{\text {planar }}=1$. The crossover between the regimes occurs sharply around $\mathrm{R} / \mathrm{H} \sim 1$. A semi-log pl ot of no-shear convex brush height, Figure 5, more clearly demonstrates that the brush expands slight, $\mathrm{H} / \mathrm{H}_{\text {planar }} \rightarrow 1.02$, near the crossover. (B) Brush grafted onto the interior of concave surface of a cylinder. For $\mathrm{R} / \mathrm{H}>10$, the brush is effectively planar as $\mathrm{H} / \mathrm{H}_{\text {planar }} \sim 1$. For the region $\mathrm{R} / \mathrm{H}<10$ bounded physically at $\mathrm{R} / \mathrm{H}=1$, the the brush shrinks with more curvature, $\mathrm{R} / \mathrm{H} \rightarrow 1$.

$\delta \mathrm{H} / \mathrm{H}_{\text {planar }} \sim \mathrm{H}_{\text {planar }} / \mathrm{R}$ and can be shown analytically from the free energy expression eq $6 .{ }^{31} \delta \mathrm{H}$ can be attributed to an increase in the volume available to each tethered chain upon stretching and is directly attributed to the Alexander-deGennes ansatz that we employ. This increase in brush height with small, convex curvature disappears with self-consistent field calculations, ${ }^{20,22}$ where the free chain ends are not constrained to lie at the brush tips. This prediction of nonzero $\delta \mathrm{H}$ is small.

For concave grafting surfaces, there exists no comparable scaling regime for nonsheared brushes. Clearly, $\mathrm{R} / \mathrm{H}=1$ is a physical bound for the problem and corresponds to the interior of the cylinder being completely filled with brush. For $\mathrm{R} / \mathrm{H}>10$, the brush is essentially planar. With increased concavity, brush height decreases to alleviate the excluded volume penalty of the more crowded tips nearer the center of the cylinder. This prediction of nonsheared concave brush height is dramatically different from the selfconsistent field results, which predict the brush height to increase with convexity. ${ }^{20}$ This is addressed later, in section IV.

\section{Shear Swelling of Convex and Concave Brushes}

Figure 4 shows characteristic swelling, given by the ratio of sheared brush height to brush height in the absence of shear, $\Delta=\mathrm{H} / \mathrm{H}_{0}$, as a function of the dimensionless shear, $\mathrm{f}$, for brushes grafted onto a planar or convex (exterior) surface of a cylinder. Here, $\mathrm{H}_{0}$ is the unsheared brush height. Note that the brush expands sharply with low shear force, $f>0$, but attains a plateau swelling at large $f$. The plateau swelling, $\Delta_{\text {max }}$, in the planar case is $2^{1 / 3}$, or about $125 \%$, but varies

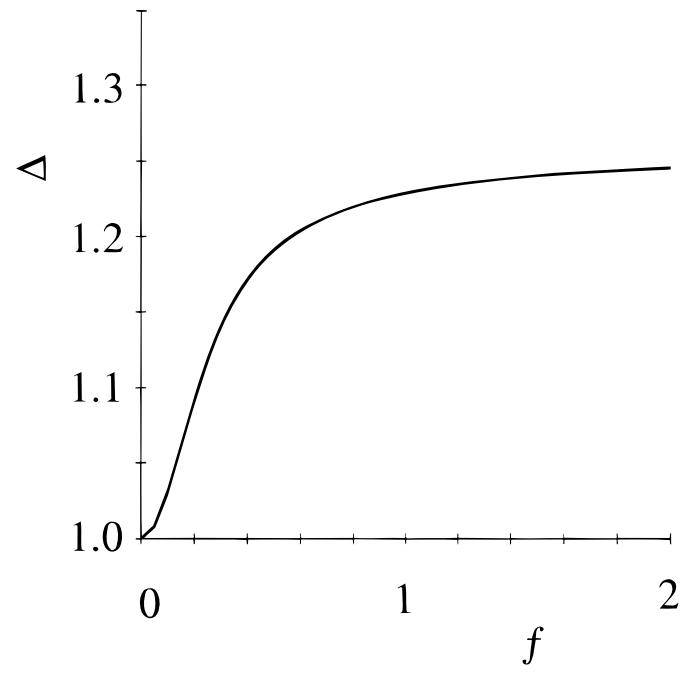

Figure 4. Characteristic shear swelling of planar brush, $\Delta$, as a function of dimensionless shearing force, $f$. For small values of an applied shearing force, the shear swelling increases as the square of the force, and at large forces, the brush sustains a maximum, plateau swelling of $\Delta_{\max }=125 \%$. Convex brushes show similar $\Delta$ versus f behavior; however, the maximal plateau swelling, $\Delta_{\max }$, differs depending on the convex curvature, $\mathrm{R} / \mathrm{H}$.

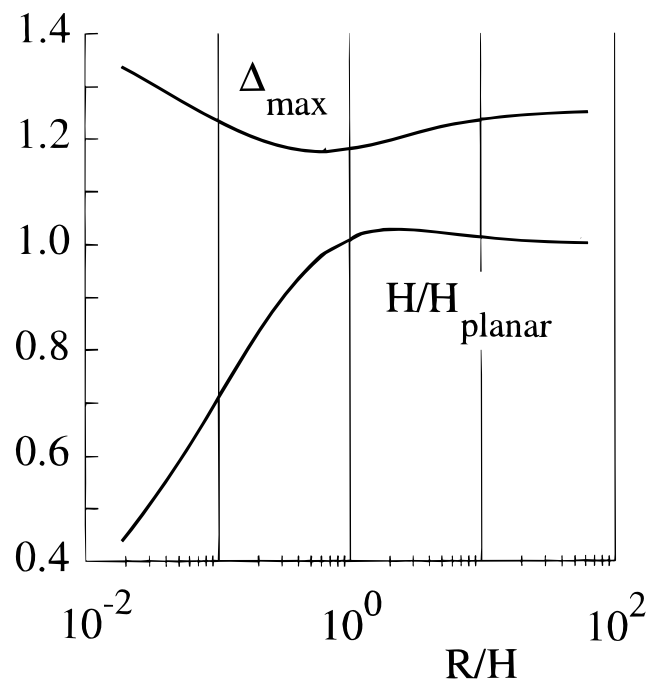

Figure 5. Convex brush height as a function of curvature, $R / H$, found from minimization of eq 6 with discretization 100 $<\mathrm{N}<500$ and grafting density $8<\mathrm{d} / \mathrm{a}<12$. (A) Maximal swelling of convex brush, $\Delta_{\max }$, versus curvature, $\mathrm{R} / \mathrm{H}$. Note that the convex brush is least shear sensitive at $R / H=1$. Maximum swelling, $\Delta_{\max }$ increases with an increase in convexity over a range of $10-15 \%$ of the nonsheared brush height. (B) Reduction in nonsheared brush height, $\mathrm{H} / \mathrm{H}_{\text {planar, }}$, with curvature R/H. The semi-log plot more clearly shows that the no-shear brush height is approximately $2-3 \%$ larger than the planar brush near $\mathrm{R} / \mathrm{H}=1$.

with convex curvature, according to Figure 5 . Figure 5 displays the maximum shear swelling, $\Delta_{\max }$, versus $\mathrm{R} / \mathrm{H}$ along with $\mathrm{H} / \mathrm{H}_{\text {planar }}$ for nonsheared convex brushes. While the brush expands at all convex curvatures (R/H $<1$ ), the maximal swelling is smallest near the crossover, $\mathrm{R} / \mathrm{H} \rightarrow 1$. This prediction of depressed swelling near the crossover might be attributed to the ansatzrelated increase in the slightly convex, nonsheared brush height. In the Alexander-deGennes treatment, the chains in a nonsheared, slightly convex brush are al ready stretched beyond the planar case; consequently, the incremental stretching penalty is larger, and the driving force for shear expansion is reduced. F or more 


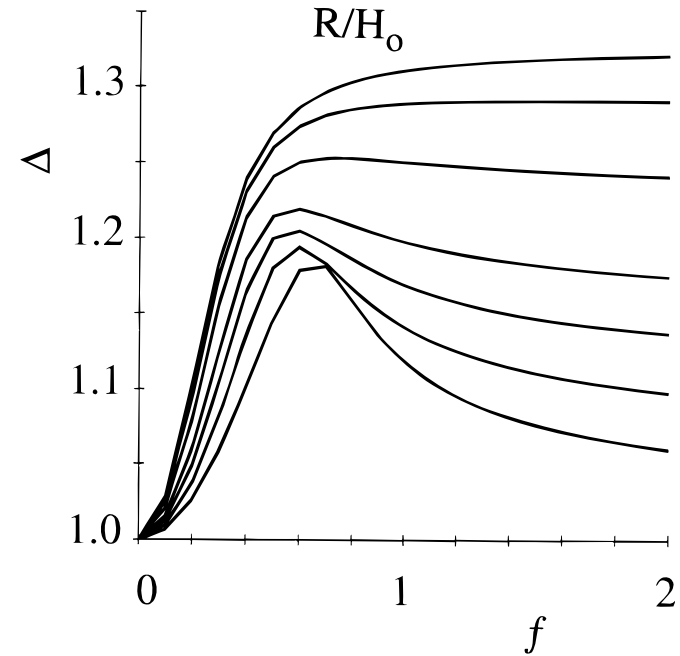

Figure 6. Shear swelling of concave brush, $\Delta$, as a function of the dimensionless shearing force, $f$, for various cylindrical radi $\mathrm{i}, \mathrm{R} / \mathrm{H}_{0}$. F or large values of $\mathrm{R} / \mathrm{H}_{0}$, the swelling versus force curves are similar to those of convex and planar brushes; however, as the cylindrical radius is decreased, the brush height becomes maximum at smaller applied force, beyond which the brush height decreases with increased shearing force. Along the contour of each line, the ratio $R / H$ is varying: for large values of $R / H_{0}$, the value of $R / H$ decreased asymptotically, attaining its minimum at the plateau swelling, while for smaller values of $R / H_{0}$, the value of $R / H$ attains minimal values in the range 1.2-1.5.

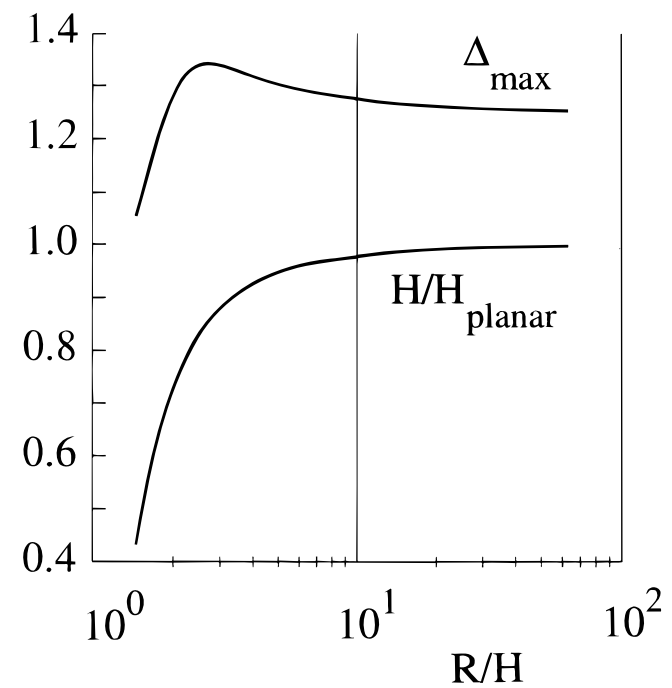

Figure 7. Concave brush height as a function of curvature, $\mathrm{R} / \mathrm{H}$, found from minimization of eq 6 with discretization 100 $<\mathrm{N}<500$ and grafting density $8<\mathrm{d} / \mathrm{a}<12$. (A) Maximal swelling of concave brush, $\Delta_{\max }$, versus curvature, $\mathrm{R} / \mathrm{H}$. Note that the maximum swelling is nonmonotonic with curvature, exhibiting maximum swelling at $\mathrm{R} / \mathrm{H}=2.3$ and decreased shear sensitivity for larger more concave surfaces. (B) Reduc-

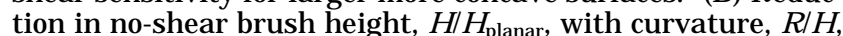
predicted using the Alexander-deGennes ansatz. SCF calculations suggest $\mathrm{H}_{0} / \mathrm{H}_{\text {planar }}>1$ for concave surfaces.

convex grafting surfaces, where the AlexanderdeGennes ansatz and the self-consistent field calculations predict identical scaling behavior, the brush swells appreciably with shear, exceeding 35\% maximum swelling. Consequently, we can expect that the maximum swelling of brushes, $\Delta_{\max }$, increases with grafting surface convexity, $\mathrm{H} / \mathrm{R}$.

The concave brush behaves differently under shear. Figure 6 shows the effect of an increasing dimensionless shear, $\mathrm{f}$, upon brush expansion, $\Delta=\mathrm{H} / \mathrm{H}_{0}$, for brushes grafted within cylinders of various radii, R. At large
Table 1. Table of Component Gradients of the Free Energy of a Grafted Brush, $\mathbf{d F}_{\mathbf{j}} / \mathbf{d H}^{\mathrm{a}}$

\begin{tabular}{lccc}
\hline surface & $\mathrm{dF}_{\text {stretch }} / \mathrm{dH}^{-}$ & $\mathrm{dF}_{\text {excluded }} / \mathrm{dH}$ & $\mathrm{dF}_{\text {shear }} / \mathrm{dH}$ \\
\hline planar & + & - & - \\
convex & + & - & - \\
concave & & + & - \\
$\mathrm{R} / \mathrm{H}<3 / 2$ & + & - & - \\
$\mathrm{R} / \mathrm{H}>3 / 2$ & + &
\end{tabular}

a The index j represents the stretching, excluded volume, and shearing components $(j=$ stretch, excluded, or shear). $A+$ sign indicates $\mathrm{dF}_{\mathrm{j}} / \mathrm{dH}>0$, i.e., the jth component of the free energy favors a decrease in brush height. A - sign indicates that the component of the energy favors increased brush height. The excluded volume component of the free energy of a concave brush favors a decrease in brush height at large curvatuve $(\mathrm{R} / \mathrm{H}<3 / 2)$ and an increase in brush height at small curvature $(\mathrm{R} / \mathrm{H}>3 / 2)$.

$R$, the effect of shear is similar to that found in convex and planar brushes, i.e., swelling increases rapidly with shear and reaches a plateau swelling, $\Delta_{\max }$. However, at smaller cylinder radii, the plateau turns into a maximum, beyond which the brush height decreases with increasing shear. The maximum in the swelling corresponds with a minimum $\mathrm{R} / \mathrm{H}$, which ranges from 0.50 to 1.5 . Figure 7 displays the shear swelling at dimensionless shear of $f=2.0, \Delta_{f=2.0}$ versus $R / H$, al ong with the nonshear height dependence on concavity. Note that the maximum swelling increases from its planar case of $125 \%$ to $135 \%$ at $\mathrm{R} / \mathrm{H}=2.3$. As curvatures increase, or R/H decreases from 2.3, the shear expansion falls to zero, and highly concave brushes show little response to shear. An increase in concavity crowds the $\mathrm{N}_{c}$ number of chain ends onto a perimeter of decreasing $r(0)$ until excluded volume energy no longer favors brushes swelling but suppresses further swelling.

\section{Discussion}

The difference in shear response of concave and convex brushes can be understood from the component gradients of a more simplified form of eq 6 ,

$$
\mathrm{F}=\mathrm{N}\left(\mathrm{F}_{\text {stretch }}+\mathrm{F}_{\text {excluded }}+\mathrm{F}_{\text {shear }}\right)
$$

where the stretching, excluded volume, and shearing energy are expressed approximately using a uniform stretching profile $|\mathrm{dx}(\mathrm{m}) / \mathrm{dm}| \rightarrow \mathrm{H} / \mathrm{N}$ :

$$
\begin{aligned}
& \mathrm{F}_{\text {stretch }}=\cos ^{-5 / 2} \theta(\mathrm{H} / \mathrm{N})^{5 / 2} \\
& F_{\text {excluded }}=W /(R+H / 2)(H / N)^{-1 / 2} \quad \text { convex } \\
& =\mathrm{W} /(\mathrm{R}-\mathrm{H} / 2)(\mathrm{H} / \mathrm{N})^{-1 / 2} \quad \text { concave } \\
& \mathrm{F}_{\text {shear }}=-\mathrm{fa} \sin \theta \mathrm{H} / \mathrm{N}
\end{aligned}
$$

Table 1 summarizes the components of the free energy gradient, $\mathrm{dF} / \mathrm{dH}$, for convex and concave brushes. Clearly, the elastic energy, $F_{\text {stretch, favors small brush }}$ heights as $\mathrm{dF}_{\text {stretch }} / \mathrm{dH}>0$. The shearing energy, $\mathrm{F}_{\text {shear, }}$ favors larger brush heights. In the convex brush, $d F_{\text {excluded }} / d H$ is negative for all values of $R / H$, so that the excluded volume and energy al ways promote brush swelling, in opposition to the chain stretching energy. However, the concave brush is different in that gradient of the excluded volume energy,

$$
\mathrm{dF}_{\text {excluded }} / \mathrm{dH}=-\mathrm{W} \sqrt{\mathrm{N}} \frac{\mathrm{R}-{ }^{3 / 2} \mathrm{H}}{\mathrm{H}^{3 / 2}(\mathrm{R}-\mathrm{H} / 2)^{2}}
$$


can be either positive or negative, favoring either smaller or larger brush heights, depending on the curvature. For $\mathrm{R} / \mathrm{H}>3 / 2, \mathrm{dF}_{\text {excluded }} / \mathrm{dH}<0$, indicating that the excluded volume interactions favor larger brush heights, while for $\mathrm{R} / \mathrm{H}<3 / 2, \mathrm{dF}$ excluded $/ \mathrm{dH}>0$, and smaller brush heights are favored. Moreover, the magnitude of this component of the free energy gradient grows as $\mathrm{R} / \mathrm{H}$ diminishes from $3 / 2$ toward 1 .

The nonmonotonic response of sheared, concave brushes can be explained by the changing sign of the gradient $\mathrm{F}_{\text {excluded. }}$ Consider a brush, subjected to a constant shearing force, but whose grafting surface concavity is variable. From the gradients in Table 1, one might expect a critical concavity, $\mathrm{R} / \mathrm{H}_{\mathrm{c}}$ smaller than $3 / 2$, for which the gradients of $F_{\text {stretch }}$ and $F_{\text {excluded }}$ balance $\mathrm{F}_{\text {shear, }}$ and neither shear expansion nor shear shrinkage of the brush is favored. Below $\mathrm{R} / \mathrm{H}_{\mathrm{c}}$, the gradient $\mathrm{dF}_{\text {excluded }} \mathrm{dH}$ will be sufficiently large and positive, rendering $\mathrm{dF} / \mathrm{dH}>0$ so that the brush height will decrease in height. Our detailed minimization, which accounts for nonuniform chain stretching, eq 6 , shows this nonmonotonic shear response where $\mathrm{R} / \mathrm{H}_{\mathrm{c}}=2.3$ (Figure 7). The more practical situation is a brush grafted to a surface of fixed concavity, where the shearing force is varied from no-shear to maximum response. If the no-shear $\mathrm{R} / \mathrm{H}$ is not too much greater than $3 / 2$, application of a small shearing force will result in brush expansion, reducing the value of $\mathrm{R} / \mathrm{H}$. If shear results in $\mathrm{R} / \mathrm{H}$ well bel ow $3 / 2$, where $\mathrm{dF}_{\text {excluded }} / \mathrm{dH}$ is large and positive, rendering $\mathrm{dF} / \mathrm{dH}>0$, then further shear will bring about brush shrinkage and an increase in $\mathrm{R} / \mathrm{H}$. The minimum in $\mathrm{R} / \mathrm{H}$ as dimensionless shear $\mathrm{f}$ is increased should occur, according to the uniform stretching profile model, Table 1 , at some value of R/H less than $3 / 2$. The detailed minimization, which accounts for nonuniform stretching, Figure 6 , shows the maximum shear swelling to occur at minimium values of $0.5<\mathrm{R} / \mathrm{H}$ $<1.5$.

The approximations that we have made in this analysis deserve comment. We assumed that the free ends of all tethered chains are localized at the brush surface and that all chains are stretched equally but not necessarily uniformly along the chain's contour. The Alexander-deGennes ansatz has been relaxed recently for the case of planar brushes ${ }^{32}$ under shear, and much the same results were obtained with and without this assumption. Thus, the Alexander-deGennes approach simplifies the analysis of planar brushes and still recovers the salient features of brush height with and without shear. However, for nonplanar brushes, the effect of curvature on the distribution of free ends of the chains is an important consideration in assessing our results. Consider an unsheared brush grafted onto a flexible surface which can be made convex, planar, or concave. Clearly, in the planar brush, there exists a distribution of free ends. As the surface is made more convex, the free ends become more local ized toward the tip of the brush, and the approximation made in the free energy analysis becomes more appropriate. In contrast, if the surface were to be made concave, we would expect a wider distribution of free ends throughout the brush height, and the approximation would be most poor in highly concave geometries. Consequently, we can expect that the incorporation of free end distribution into the energy analysis will affect mostly our predictions of concave brushes and will minimally affect our predictions for convex brushes. While the distribution of free ends can be measured in nonsheared planar brushes using neutron reflectometry, or calculated for nonsheared convex and planar brushes using self-consistent field approach, there exists no exact calculation of the free end distribution in concave geometries or, more importantly, no experimental determination of how shearing of the brush affects the distribution of free ends.

\section{Conclusions}

In summary, we have performed the first free energy analysis for the shear response of brushes on nonplanar surfaces using the ansatz that all chains possess the same nonuniform stretching profile with chain ends localized at the brush surface. Our results predict that the response of a brush to shear in a good solvent depends on the curvature of the grafting surface. Convex brushes expand with an applied shear, swelling upwards of $35 \%$ of their nonsheared brush height, with maximal swelling increasing as convexity increases, for $\mathrm{H} / \mathrm{R}<1$. Thus, highly convex brushes are predicted to swell more than planar brushes (25\%) of the same grafting density. In contrast, highly concave brushes exhibit nonmonotonic swelling with shear: they swell to a maximal value, followed by a reduction of brush height with increased shear. Brushes which are slightly concave exhibit a higher maximum shear swelling than planar brushes of the same grafting density; however, with increased concavity, the predicted response of the brush to shear is much reduced.

The response of nonplanar brushes can be important to a number of applications. The shear response of a concave brush is an important feature in porous flow applications. According to the shear response described here, membrane pores lined with a polymer brush will possess a range of effective diameters, depending on the prevailing shear at the brush or the applied pressure drop. Consequently, a membrane whose pores are lined with a polymer brush will be dynamic, showing filtration and flow control that depends on the applied pressure drop. Indeed, for slitlike pores, nonlinear volumetric flow rates with applied pressure are predicted, ${ }^{6}$ indicating the potential for polymer brush microvalves which require no mechanical parts or control loops. The response of a convex brush to shear is important in the rheology of, for example, hairy-rod polymers, mol ecular bottlebrushes, or brush-covered particulates. The size of the molecule with brushlike architecture depends on the expansion of the brush lining the surface that is in shear.

Acknowledgment. E.M.S. thanks David Williams for discussions and acknowledges financial support from the National Science Foundation NSF-CAREER Award while at the Department of Chemical Engineering, University of Colorado at Boulder.

\section{References and Notes}

(1) Klein, J .; Perahai, D.; Warburg, S. Nature 1991, 352, 143.

(2) Klein, J. Colloids Surf. A 1994, 86, 63.

(3) Barrat, J .-L. Macromolecules 1992, 25, 832.

(4) Rabin, Y.; Alexander, S. Europhys. Lett. 1980, 13, 1069.

(5) Kumaran, V. Macromolecules 1993, 26, 2464.

(6) Sevick, E. M.; Williams, D. R. M. Macromolecules 1994, 27, 5285.

(7) Sevick, E. M.; Williams, D. R. M. Porous membranes with grafted polymers: valves and sensors. In Materials for Smart Systems; George, E. P., Takahashi ,S., Trolier-McKinstry, S., Ulchino, K., Wun-Fogle, M., Eds.; MRS Symposia Proceedings 360; Materials Research Society: Pittsburgh, PA, 1995. 
(8) Sevick, E. M.; Williams, D. R. M. Pressure-sensitive microvalves made from polymer brushes. In Advances in Porous Materials; Komarneni, S., Smith, D. M., Beck, J. S., Eds.; MRS Symposia Proceedings 316; Materials Research Society: Pittsburgh PA, 1995.

(9) Harden, J. L.; Cates, M. E. J Phys. II 1995, 5, 1093.

(10) Schmidt, A.; Lehmann, S.; Georgelin, M.; Katana, G.; et al. Macromolecules 1995, 28, 5487.

(11) Fredrickson, G. H. Macromolecules 1993, 26, 2825.

(12) Tsukahara, Y.; Tsutsumi, K.; Yamashita, Y.; Shimada, S. Macromol ecules 1990, 22, 1546.

(13) Wintermantel, M.; Gerle, M.; Fischer, K.; Schmidt, M.; Wataoka, I.; Urakawa, H.; Kajiwara, K.; Tsukahara, Y. Macromol ecules 1996, 29, 978.

(14) Alexander, S. J . Phys. (Paris) 1977, 38, 983; deGennes P.-G. J. Phys. (Paris), 1976, 37, 1443.

(15) Daoud, M.; Cotton, J. J . Phys. 1982, 43, 531.

(16) Birshtein, T. M.; Borisov, O. V.; Zhulina, Y. B.; Khokhlov, A. R.; Yurasov, T. A. Polym. Sci. USSR 1987, 29, 1293.

(17) Toral, R.; Chakrabarti, A. Phys. Rev. E 1993, 47, 4240.

(18) Murat, M.; Grest, G. S. Macromolecules 1991, 24, 704.

(19) Semenov, A. N. Sov. Phys. J ETP 1985, 61, 733.
(20) Milner, S. T.; Witten, T. A. J . Phys. (Paris) 1988, 49, 1951.

(21) Ball, R. C.; Marko, J. F.; Milner, S. T.; Witten, T. A. Macromolecules 1991, 24, 693.

(22) Dan, N.; Tirrell, M. Macromolecules 1992, 25, 2890.

(23) Karim, A.; Satija, S. K.; Douglas, J . F.; Ankner, J . F.; Fetters, L. J . Phys. Rev. Lett. 1994, 73, 3407.

(24) Auroy, P.; Mir, Y.; Auvray, L. Phys. Rev. Lett. 1992, 69, 93.

(25) Lai, P.-Y.; Binder, K. J . Chem. Phys. 1993, 98, 2366.

(26) Peters, G. H.; Tildesley, D. H. Phys. Rev. E 1995, 52, 1882.

(27) Williams, D. R. M. Macromolecules 1993, 26, 373.

(28) Pincus, P. A. Macromolecules 1976, 9, 386.

(29) de Gennes, P. G. Scaling Concepts in Polymer Physics; Cornell University Press: Ithica, NY, 1979.

(30) Hillstron, K. Nonlinear Optimization Routines in AMDLIB Technical Memorandum No. 297; Applied Mathematics Division, Argonne National Laboratory, Argonne, IL, 1976; Subroutine GQBFGS in AMDLIB.

(31) Sevick, E. M.; Williams, D. R. M. Submitted to J . Chem. Phys.

(32) Harden, J. L., personal communication.

MA9604552 